\title{
Impact of Network Topology on Energy Efficiency in WSN
}

https://doi.org/10.3991/ijoe.v17i09.23089

\author{
Mohammed Réda El Ouadi $\left({ }^{凶}\right)$, Abderrahim Hasbi \\ Mohammed V University, Rabat, Morocco \\ elouadi.mr@gmail.com
}

\begin{abstract}
Wireless communication network has a significant success in scientific and industrial communities. Due to its various advantages, this technology is considered as a key element in current network architectures. It represents the architecture that allows to group a large number of sensors to collect information about a physical process in different environments. The gathered data is transmitted to base station which communicates the information to the end user. Several protocols are proposed for WSNs routing, by considering the limited capacities of sensor nodes according to a specific topology that allows to organize the nodes within the network. However, the performance of each routing protocol mainly depends on the application requirements and its results in terms of the lifetime of WSN and satisfaction of objectives defined. According of the structure of WSN, the routing techniques can be divided in three types hierarchical, location-based flat routing. This paper, present the different routing techniques in WSN, based on the organization of nodes in sensor area. We focus specially to study the three types, cluster-based, chain-based and location-based routing techniques. These techniques will be simulated in order to compared their performances with our protocol Location-Based LEACH (LOCLEACH).
\end{abstract}

Keywords-WSN, LEACH, Clustering, routing, energy consumption

\section{Introduction}

Wireless sensor networks (WSN) consist of grouping sensor nodes together to cooperate and collect data from a specific environment [1]. The gathered data from all nodes is supplied to a centralized node, called a base station (BS). The BS is responsible to analyze data, making decision and diffuse data for user usage through Internet [2]. WSN can be deployed in different domains and environments to monitor and analyze a physical event, such as military intervention, health domain and Internet of things (IOT) applications.

Military applications represent the first field using the concept of wireless sensor networking [3]. WSN are generally used in military operations, to monitor a military or border zone and targeting systems [3]. WSN represent a promising concept in mili- 
tary domain, due to its characteristics in terms of fault tolerance, Self-organization and rapid deployment.

In medical domain, WSN play an important role to improve the healthcare conditions, by proposing an efficient tool to monitor and analyze the physiological condition of patients [4]. These sensors are placed directly on the patient's body to detect each dysfunction and transmit the information's urgently to the appropriate medical unit (oxygen saturation, temperature, heart attack, blood pressure, etc.) [4] [5]. The concept of IoT consists to connect identifiable devices within the internet connection for sensing and monitoring tasks [6]. Thus, it represents an attractive domain of technological advance. The WSN assures communications amongst numerous low-power IOT objects or nodes using various protocols [6] [7].

The organization of nodes in the network in a uniform and optimal way allows to extend the duration of the network [2]. The routing protocol specifies the distribution of nodes into the network, the size of clusters and how to manage each change concerning the organization of nodes [8]. Therefore, the choice of the topology of the network represent a big challenge in WSN. It depends on several parameters such as: domain application of WSN, numbers of sensor nodes, distance of Base station.

The most popular topologies of WSNs are presented in this paper: Clustered based, Chain based and location based concepts, in order to compare them and analyze the impact of wsn topologies on network lifetime and energy consumption. Furthermore, the objective of this work is to evaluate our LOC-LEACH protocol, by comparing it with other routing protocol using different concepts of topologies.

In location-based protocols, location of nodes and distances between them, are used to select the optimal path to transmit data with minimum energy consumption [9]. The chain routing concept, organizes sensor nodes in chain form, and each node transmits data to its close neighbor until arrived to BS [10]. The clustered based routing consists to group nodes into clusters, respecting to specific metrics. The clusters are composed of same ordinary nodes and one of them called $\mathrm{CH}$; is responsible to gather data from all node in the cluster and transmit an aggregated packet to the BS, of many ordinary nodes and one leader node named cluster head $(\mathrm{CH})$. The $\mathrm{CH}$ is considered the most crucial component to manage in the hierarchical routing protocol.

This paper is divided in three parts. First, we study the classification of routing protocols in WSN. The second part of this paper presents a related work study of three topologies in WSN. In the end, different protocols using the three topologies will be compared with LOC-LEACH protocol [11].

\section{Related work}

Li Qing et Al propose an enhanced version of LEACH protocol named DEEC (Distributed Energy Efficient Clustering). The main objective is to prolong the lifetime of WSN by giving more chances to nodes to become a $\mathrm{CH}$. The $\mathrm{CH}$ is chosen according to ratio of network average energy and residual energy of node. DEEC defines two status of node in the two-level heterogeneous networks, normal and advanced nodes. The status of each node is characterized by its amount of energy. The 
number of round when the node can be elected $\mathrm{CH}$ is based of its amount of energy. In DEEC, nodes with more energy are favored to be elected $\mathrm{CH}$ than other nodes. The authors of [12] present many enhancements that improve the limits of DEEC. Developed DEEC (DDEEC), Enhanced DEEC (EDEEC) and Threshold DEEC (TDEEC) are simulated to evaluate and compare their performances. The results show that TDEEC performs better in terms of the lifetime of WSN and stability period but instability period of EDEEC and TDEEC is very large. EDEEC and TDEEC improve the period of stability of network while compromising on lifetime.

Another improvement of LEACH proposed by Power-efficient gathering in sensor information systems (PEGASIS) protocol [13]. The protocol uses Chain based concept and nodes are organized in chain. One node of these chain called leader, responsible to transmit aggregated data from all nodes to the BS. The role of leader is taken in turn by the different nodes, according to a selection procedure defined by the protocol. The greedy algorithm is used to organize the chain and each node must have total knowledge of the network. Collaboration technique is used between nodes allows to minimize traffic of data transmission in the network and prolong network lifetime. An improvement of PEGASIS is proposed in [14] named Energy efficient PEGASIS based protocol (EEPB). It adopts distance threshold when constructing chain, to decline the long links in PEGASIS. Thus, the leader is selected by considering its amount of energy and the distance that separates it from the base station. The improved energy efficient PEGASIS protocol (IEEPB) [14] performs EEPB, by modifying the process of chain construction. it proposes a new process to form chain and remove the long link problems using threshold computations. Mohsin Raza Jafri et al propose a new improvement protocol named Mobile sink improved energy-efficient PEGASIS-based routing protocol (MIEEPB) [14]. It uses a mobile base station to gather data from all nodes, and divides network into smaller chains. The main objective is to minimize data delivery delay and to reduce the distance between connected nodes through multi chains concept. The protocol presents how these concepts can affect largely in enhancing the network lifetime of wireless sensors. Using the simulation, MIEEPB shows a best result than PEGASIS and its enhanced versions (PEEB) and (IPEEB).

In [8], authors studied LEACH and PEGASIS in order to compare chain-based and cluster-based mechanisms. They conclude that PEGASIS performs better than LEACH in terms of communication overhead and network lifetime. In [15], Q. Nadeem et al present gateway based energy-efficient routing protocol (M-GEAR) that uses a Location-Based concept. M-GEAR divides the network in four logical zones according to location of nodes in the sensor area. -Each region elects its own $\mathrm{CH}$ independently of other regions. Simulation results shows that M-GEAR allows high performance than LEACH in terms of network life time and energy consumption. Best results were obtained by our LOC-LEACH protocol in [11]. It divides the network into different regions based on the distance of nodes from the base station. The nodes close to the $\mathrm{BS}$, form the first region, and a direct transmission to the $\mathrm{BS}$ is adopted. The other nodes are organized into two regions using the clustering concept, and adopting a new $\mathrm{CH}$ selection algorithm based on the energy of the nodes of each 
region. LOC-LEACH is an improved version of the LEACH protocol to improve network life and energy efficiency (Figure 1).



Fig. 1. LOC-LEACH: Protocol topology

\section{Simulation results}

In this section we discuss simulations results of the main protocols presented in this paper and compare them which LOC-LEACH protocol. the simulation is used through Matlab simulator. In our simulation, we consider:

- WSN with 100 nodes distributed randomly in $100 \mathrm{~m} \times 100 \mathrm{~m}$ field,

- Sensor nodes are homogeneous and with same characteristics in terms of computing energy, and sensing.

Parameters for our simulation are given in the Table 1.

Table 1. Parameters of the simulation

\begin{tabular}{|l|c|}
\hline \multicolumn{1}{|c|}{ Parameter } & Value \\
\hline Network size & $100 \mathrm{~m} * 100 \mathrm{~m}$ \\
\hline Initial Energy & $0.5 \mathrm{~J}$ \\
\hline Base station location in stationary cases & 50,120 \\
\hline Data Aggregation Energy cost & $5 \mathrm{pJ} / \mathrm{bit}$ \\
\hline Number of nodes & 100 \\
\hline Packet size & $4000 \mathrm{bit}$ \\
\hline transmission energy Eelec & $5 \mathrm{~nJ} / \mathrm{bit}$ \\
\hline Reception energy Efs & $10 \mathrm{pJ} / \mathrm{bit} / \mathrm{m} 2$ \\
\hline Transmit amplifier (Eamp) & $0.0013 \mathrm{pJ} / \mathrm{bit} / \mathrm{m} 4$ \\
\hline
\end{tabular}




\subsection{Alive nodes}

In MIEEPB, the stability period is until 1500 rounds, it represents the double of result obtained by M-GEAR (750 rounds), $600 \%$ of DEEC results (250 rounds), and the same results than LOC-LEACH (1500 rounds). On other hand, MIEEPB performs the both protocols DEEC and M-GEAR in terms of network lifetime. It prolongs the death of nodes throughout the lifecycle of the network $(10 \%, 50 \%$ and $100 \%$ of nodes). In MIEEPB, The last node death until the 4500 rounds, 2500 for M-GEAR, 3100 for DEEC and 2300 for LOC-LEACH. MIEEPB improves the PEGASIS protocol by dividing the long chain and allowing the mobility of the sink. It improves significantly the results of the chain-based concept compared to the improvements of the other concepts (cluster-based and location-based). In [24] MIEEPB has also performed well compared to other chain based protocols and especially with the classical PEGASIS. In terms of stability of stability period, our proposed protocol LOCLEACH is the only one gives the same result than MIEEPB, however the lifetime of WSN can be enhanced to achieve better result (see Figure 2).
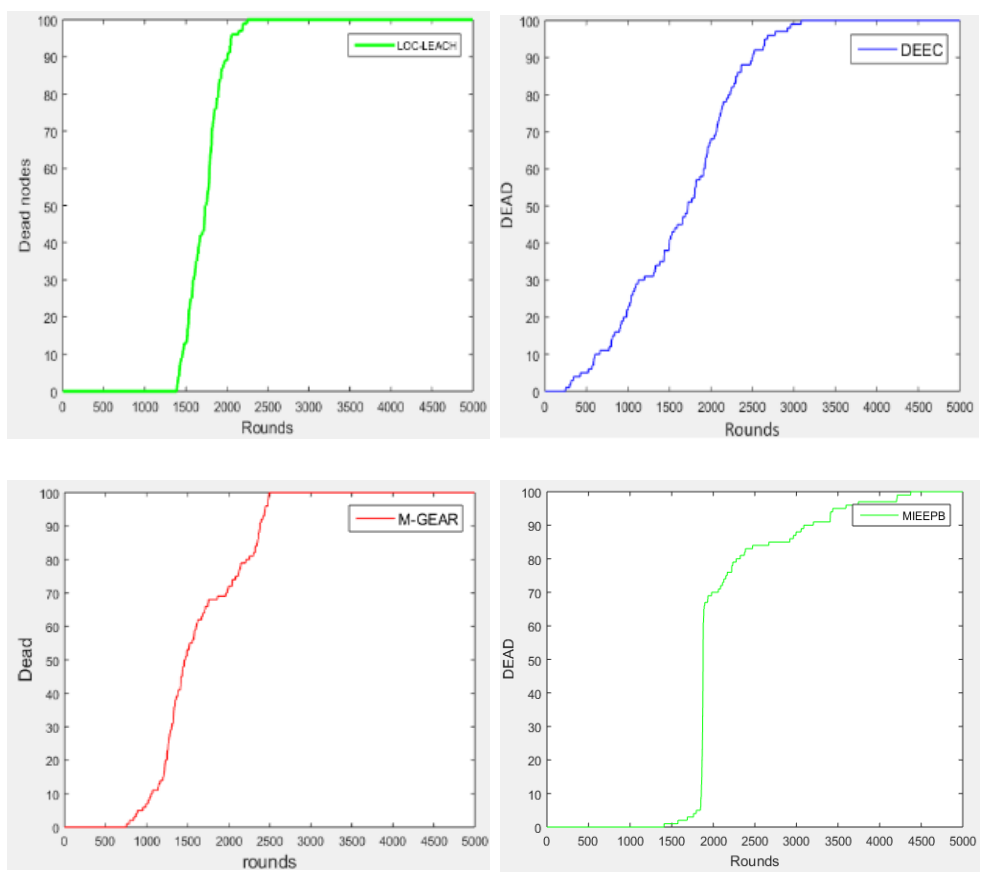

Fig. 2. Comparison of Alive nodes

\subsection{Energy consumption}

The Figure 3 shows that MIEEPB conserve more energy than DEEC and LOC$\mathrm{LEACH}$, due of its nodes organization in multi chain, and mobility of the sink. These 
improvements allow an optimal distance between BS and nodes and take a better result from the energy consumption.
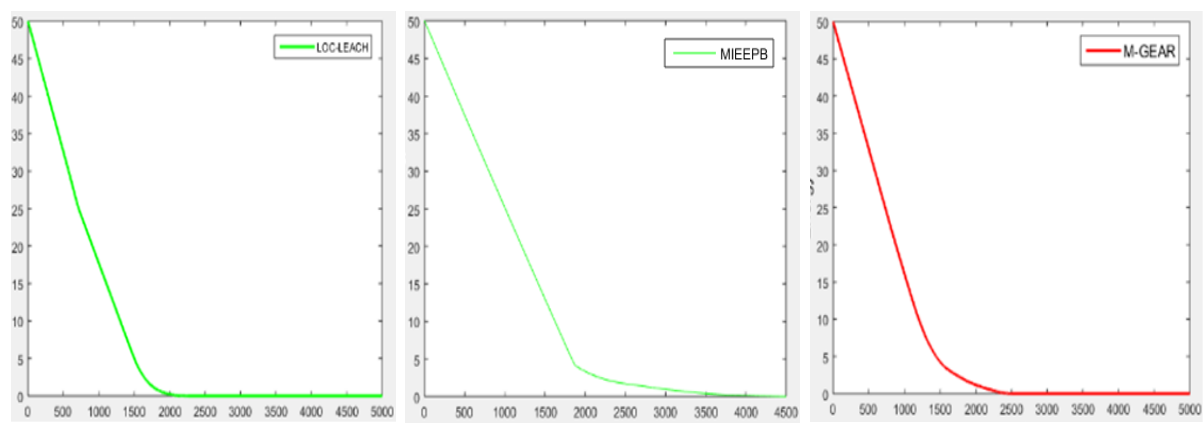

Fig. 3. Energy consumption of M-GEAR, MIEEPB and LOC-LEACH

\section{Discussion}

In this section, we are interested to compare our protocol LOC-LEACH with the MIEEPB protocol in terms of the lifetime of WSN and repartition of death nodes. The choice of the MIEEPB for comparison is based on their good performances compared of other routing protocols and their improvement.

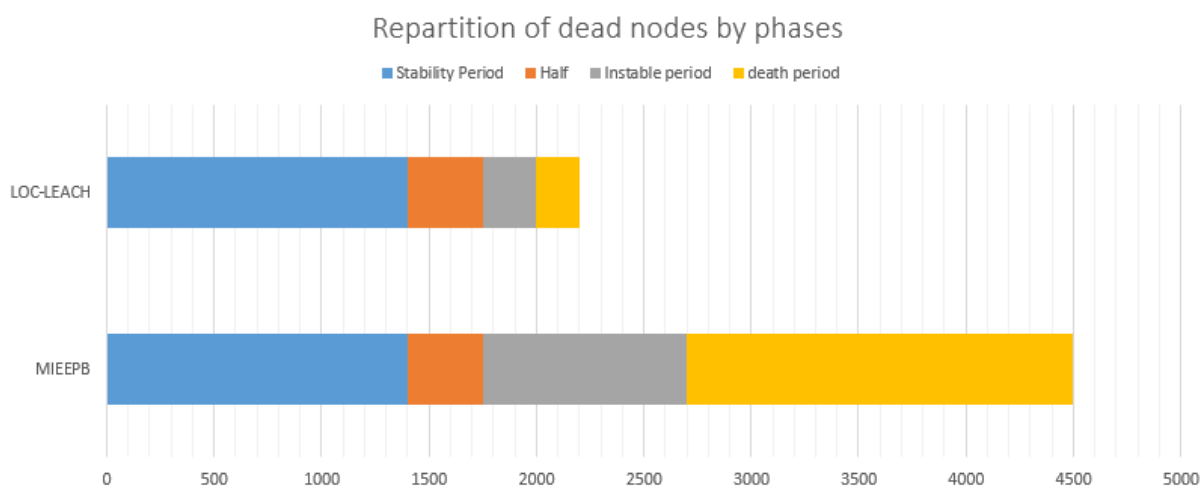

Fig. 4. Repartition of dead node in LOC-LEACH and MIEEPB

The figure represents the result of this comparison by dividing the network life cycle in phases. The stability period that represents the period where the network keeps its initial topology before the death of the first node. The second phase represents when the network loses $50 \%$ of the initial number of nodes. After that, we found the period when the network works with $50 \%$ until $90 \%$ of its initial nodes, and the last period is the moment when $90 \%$ of nodes are dead and the network keeps just $10 \%$ of nodes for perform all tasks. 
Protocols have the same results during the both first periods until the network works with $50 \%$ of its initial topology. The first two periods of routing protocol represent the most important periods in terms of data performance and reliability, when all regions of network are covered and transmit data with maximum of reliability.

MIEEPB performs our protocol LOC-LEACH in the 2 others periods and specially in the last one, when the network continues to perform its functioning with the minimum alive nodes (10\% of nodes). MIEEPB can keep the network alive with this minimum node alive 2300 rounds than LOC-LEACH. This may benefit for small networks that require no total network coverage. However, for large networks this period with just $10 \%$ of alive nodes may be irrelevant since the data collected does not represent the real situation, and in this case the network may be considered death.

\section{Conclusion}

In this paper different topology used for designing in WSN are presented. The main objective is to present the role of WSN topologies in prolong network lifetime and minimizing energy consumption. The performance of different routing protocols using these topologies are also discussed. Clustered based, chain based and location based topologies are analysed in this work. The analysis shows that MIEEPB protocol using chain based concept, performs better than others protocols using others concept like cluster or location based routing mechanisms in terms of network lifetime, and energy consumption. furthermore, our protocol LOC-LEACH proposed in [11] is compared with MIEEP, result prove that LOC-LEACH presents interesting performances, however, some limitations need to be improved, especially in terms of prolonging network lifetime when the network works with a minimum of alive nodes.

\section{References}

[1] G.Dhand,S.S.Tyagi, " Data aggregation techniques in WSN:Survey", ICCC, 2016

[2] Faouzi Hidoussi et al, " PEAL: Power Efficient and Adaptive Latency Hierarchical Routing Protocol for Cluster-Based WSN", Wireless Pers Commun (2017). https://doi.org /10.1007/s11277-017-4963-Z

[3] Tareq Alhmiedat, Anas Abu Taleb, Mohammad Bsoul," A Study on Threats Detection and Tracking Systems for Military Applications using WSNs", IJCA, February 2012. https://doi.org/10.5120/5055-7347

[4] Nayif saleh, abdallah kassem and ali m. Haidar," Energy-Efficient Architecture for Wireless Sensor Networks in Healthcare Applications", IEEE Access, 2018. https://doi.org /10.1109/access.2018.2789918

[5] Maria de los Angeles Cosio Leon,Jesus Luna Garcia, "A Security and Privacy Survey for WSN in e-Health Applications",Electronics, Robotics and Automotive Mechanics Conference,2009. https://doi.org/10.1109/cerma.2009.47

[6] Karimunnisa Begum,Sunanda Dixit,"Industrial WSN using IoT: A Survey"International Conference on Electrical, Electronics, and Optimization Techniques (ICEEOT) - 2016. https://doi.org/10.1109/iceeot.2016.7755660 
[7] Khalid Haseeb et al, " An Energy Efficient and Secure IoT-Based WSN Framework: An Application to Smart Agriculture", sensors, 2020. https://doi.org/10.3390/s20072081

[8] Mohammed Réda El Ouadi, Abderrahim Hasbi, "Comparison of LEACH and PEGASIS Hierarchical Routing Protocols in WSN", International Journal of Online and Biomedical Engineering (iJOE),2020. https://doi.org/10.3991/ijoe.v16i09.14691

[9] Jitender Grover, Shikha, Mohit Sharma," Location Based Protocols in Wireless Sensor Network - A Review", Fifth (ICCCNT);2014. https://doi.org/10.1109/iccent.2014.6962 $\underline{990}$

[10] Shalli Rania, Jyoteesh Malhotrab, Rajneesh Talwarc,"Energy efficient chain based cooperative routing protocol for WSN", Applied Soft Computing 35 (2015) 386-397. https://doi. org/10.1016/j.asoc.2015.06.034

[11] Hiu Fai Chan et al, " Hierarchical Routing Protocols for Wireless Sensor Network: A Compressive Survey", Wireless Networks, 2020

[12] Mohammed Réda El Ouadi, Abderrahim Hasbi," LOC-LEACH: An enhanced LEACH protocol for WSNs", International Journal of Advanced Trends in Computer Science and Engineering (IJATCSE), 2020. https://doi.org/10.30534/ijatcse/2020/55942020

[13] L T. N. Qureshi, N. Javaid, M. Malik, U. Qasim‡, Z. A. Khan,"On Performance Evaluation of Variants of DEEC in WSNs ", Seventh International Conference on Broadband, Wireless Computing, Communication and Applications,2013. https://doi.org/10.1109/bwcca .2012 .35

[14] Stephanie Lmdsey and Cauligi S. Raghavendra,"PEGASIS: Power-Efficient Gathering in Sensor Information Systems", Proceedings, IEEE Aerospace Conference,2002. https://doi .org/10.1109/aero.2002.1035242

[15] P. Aya Ayad Hussein1, RajaaaldeenAbd Khalid, " Improvements of PEGASIS Routing Protocol in WSN", International Advance Journal of Engineering Research (IAJER),2019

[16] Jafri, et al," M-GEAR: Gateway-Based Energy-Aware Multi-Hop Routing Protocol for WSNs",Eighth BWCCA, 2013. https://doi.org/10.1109/bwcca.2013.35

\section{Authors}

Mohammed Réda El Ouadi is with Mohammed V University, Mohammadia School of Engineers, Rabat, Morocco

Abderrahim Hasbi is with Mohammed V University, Mohammadia School of Engineers, Rabat, Morocco

Article submitted 2021-04-03. Resubmitted 2021-05-04. Final acceptance 2021-05-05. Final version published as submitted by the authors. 